\title{
Beyond Susceptible and Resistant, Part III: Treatment of Infections due to Gram-Negative Organisms Producing Carbapenemases
}

\author{
Navaneeth Narayanan, PharmD, ${ }^{1}$ Linda Johnson, PharmD, ${ }^{2}$ and Conan MacDougall, PharmD, MAS \\ ${ }^{1}$ Ernest Mario School of Pharmacy, Rutgers University, New Brunswick, New Jersey; ${ }^{2}$ CHI Memorial Hospital, \\ Chattanooga, Tennessee; ${ }^{3}$ Department of Clinical Pharmacy, University of California, San Francisco, School of Pharmacy, \\ San Francisco, California
}

\begin{abstract}
Carbapenemases are enzymes that are capable of inactivating all or almost all beta-lactam antimicrobial agents. These enzymes are frequently coexpressed with other resistance mechanisms to non-beta-lactams, leading to extremely drug-resistant pathogens. Once a curiosity, these enzymes have spread into organisms that are among the most common causes of infection, such as Klebsiella pneumoniae and Escherichia coli. Identification of these organisms has proved challenging for clinical microbiology laboratories, leading to revisions in susceptibility standards for carbapenems. Although currently a rare cause of infection in children, these carbapenem-resistant Enterobacteriaceae (CRE) are becoming endemic in a variety of healthcare settings. Management of infections due to CRE is complicated by a lack of effective treatment options and clinical data on their effectiveness. Treatment of CRE infections in children is particularly challenging because therapeutic options for CRE lack adequate data on dosing and safety in children. Use of unconventional combination treatment regimens, including agents to which the organism is resistant in vitro, may provide some benefit in the treatment of severe CRE infection. Fortunately, several agents with the potential for treatment of CRE infections have been recently approved or are in late clinical development, although few data will be available in the short term to inform use in children.
\end{abstract}

INDEX TERMS: beta-lactamases, drug resistance, Escherichia coli, Klebsiella pneumoniae, microbial

J Pediatr Pharmacol Ther 2016;21(2):110-119

\section{INTRODUCTION}

By far the most commonly used class of antibacterial agents used in children includes the beta-lactams, accounting for almost three-quarters of all antibacterial use. ${ }^{1}$ Acquired (as opposed to intrinsic) beta-lactam resistance among Gramnegative bacteria is typically mediated through production of enzymes that hydrolyze the betalactam molecule-beta-lactamases. Among the most concerning types of beta-lactamases to emerge in recent years are the carbapenemases. These enzymes are capable of hydrolyzing all or almost all beta-lactam antibacterials, are unaffected by most beta-lactamase inhibitors (BLIs), and have shown a predilection for epidemic spread. ${ }^{2}$ The Centers for Disease Control and Prevention recently classified carbapenem-resistant Enterobacteriaceae (CRE) as an urgent public health threat in the United States, requiring ag- gressive action. ${ }^{3}$ However, methods for detection and classification of these organisms by clinical microbiology laboratories have evolved rapidly, potentially increasing risks for inadequate detection and treatment. In this article we will review the microbiology and epidemiology of the most clinically relevant organisms producing carbapenemases and discuss the implications for antibacterial therapy in children.

\section{MICROBIOLOGY AND EPIDEMIOLOGY}

The first carbapenem was developed and approved for use in 1985 in response to the growing concern of resistance, especially among Gram-negative rods. ${ }^{4}$ Resistance among these organisms is primarily the result of the spread of beta-lactamase enzymes capable of inactivating most penicillins and cephalosporins. ${ }^{5}$ Carbapenems offered greater stability against 
Table 1. Comparison of Carbapenemases

\begin{tabular}{|c|c|c|c|c|}
\hline & \multicolumn{4}{|c|}{ Carbapenemases } \\
\hline & IMP, VIM & NDM & OXA & KPC \\
\hline Molecular class & & & $\mathrm{D}$ & A \\
\hline Functional group & & & $2 \mathrm{df}$ & $2 f$ \\
\hline \multicolumn{5}{|l|}{ Hydrolytic activity versus $\beta$-lactams* } \\
\hline Penicillins & + & + & + & + \\
\hline First- and Second-generation cephalosporins & + & + & + & + \\
\hline Third- and fourth-generation cephalosporins & + & + & \pm & + \\
\hline Carbapenems & + & + & \pm & + \\
\hline Aztreonam & - & - & - & + \\
\hline \multicolumn{5}{|l|}{ Inhibition profilet } \\
\hline Clavulanate, sulbactam, tazobactam & - & - & \pm & \pm \\
\hline Avibactam & - & - & \pm & + \\
\hline EDTA & + & + & - & - \\
\hline
\end{tabular}

EDTA, ethylenediamine tetraacetic acid; IMP, active on imipenem; KPC, Klebsiella pneumoniae carbapenemase; NDM, New Delhi metallobeta-lactamase; OXA, oxacillin-hydrolyzing; VIM, Verona integron-encoded metallo-beta-lactamase

$*+=$ strong hydrolytic activity; $\pm=$ variable hydrolytic activity; $-=$ weak hydrolytic activity

$\dagger+=$ strong enzyme inhibition; $\pm=$ variable enzyme inhibition; $-=$ weak enzyme inhibition

these beta-lactamases; however, within a few years carbapenemases, which are capable of hydrolyzing almost all beta-lactams, including carbapenems, were identified. Carbapenemases can be located on plasmids or chromosomes and, like the extended-spectrum beta-lactamases (previously reviewed in the series 6 ), are always expressed, even in the absence of their substrate. ${ }^{2}$

Carbapenemases, like the other beta-lactamases, are generally classified into functional groups and molecular classes. Under the functional group system developed by Queenan and Bush, ${ }^{2}$ carbapenemases fall within groups $2 \mathrm{f}, 2 \mathrm{df}$, and 3. Under the molecular class scheme developed by Ambler and colleagues, carbapenemases belong to classes A, B, and D. ${ }^{2}$ At present, there are 2 primary types of carbapenemases reported in Enterobacteriaceae: metallo-beta-lactamases (MBLs) and serine beta-lactamases. The MBLs belong to functional group 3a and molecular class B. These beta-lacatamases acquired their name from the presence of a zinc ion at its active site, involved in hydrolysis of the beta-lactam ring. As a result of this zinc dependency, the metal ion chelator ethylenediamine tetraacetic acid (EDTA) is able to inhibit MBL activity, and, thus, inhibition by EDTA is one means by which the presence of this enzyme is determined. In contrast, traditional BLIs such as clavulanate do not affect MBL activity. Metallo-beta-lactamases are capable of hydrolyzing all beta-lactams except aztreonam (Table 1). ${ }^{2}$ However, coproduction of other beta-lactamases, such as AmpCs and extended-spectrum beta-lactamases, often hinders the use of aztreonam. ${ }^{7}$ The most prevalent MBLS are the IMP (active on imipenem), VIM (Verona integron-encoded metallo-beta-lactamase), and New Dehli metallo-beta-lactamase (NDM) types. ${ }^{8}$ Serine beta-lactamases, the second type of carbapenemases, utilize serine at their active site and are weakly inhibited by traditional BLIs (clavulanic acid, sulbactam, and tazobactam). These enzymes are capable of hydrolyzing all beta-lactams including aztreonam (Table 1). Although weakly inhibited by beta-lactamase inhibitors in vitro, the current Food and Drug Administration (FDA)-approved beta-lactambeta-lactamase inhibitor (BLBLI) combinations are not considered to be clinically effective against carbapenemase-producing organisms. ${ }^{2}$ However, avibactam, a novel BLI, has broader inhibitory activity, and the combination agent ceftazidime/avibactam, the newest BLBLI, is promising against oxacillin-hydrolyzing (OXA48) carbapenemases and Klebsiella pneumoniae carbapenemases (KPCs), ${ }^{9-11}$ which are the most prevalent carbapenemases in North America and will be the primary subject of this article. ${ }^{12}$ 
Table 2. Clinical Laboratory Standards Institute (CLSI) Recommendations for Routine Identification of Carbapenemases ${ }^{14,16}$

\begin{tabular}{|c|c|c|}
\hline & Prior to $2010, \mathrm{mg} / \mathrm{L}$ & Current, mg/L \\
\hline \multicolumn{3}{|l|}{ Cutoff for susceptibility in Enterobacteriaceae } \\
\hline Doripenem & N/A & $\leq 1$ \\
\hline Ertapenem & $\leq 2$ & $\leq 0.5$ \\
\hline Imipenem & $\leq 4$ & $\leq 1$ \\
\hline Meropenem & $\leq 4$ & $\leq 1$ \\
\hline Phenotypic test recommended to accompany MIC testing & MHT & None \\
\hline
\end{tabular}

MHT, Modified Hodge Test; MIC, minimum inhibitory concentration; NA, not available

Although all carbapenemases have the capacity to hydrolyze carbapenems, the rate and extent of hydrolysis vary, resulting in a range of susceptibilities from a fully susceptible to a highly resistant isolate. There are several factors thought to contribute to this variability, including the amount of carbapenemase that is produced, the specific carbapenem tested, the organism involved, and the coexistence of other mechanisms of resistance, such as porin loss, efflux pumps, and the expression of other beta-lactamases. OXA-48 carbapenemases typically have the lowest minimum inhibitory concentrations (MICs) to carbapenems and, consequently, are the hardest to identify, followed by MBLs and lastly KPCs, which have the highest MICs. ${ }^{13}$

Carbapenemases were particularly difficult to identify using the Clinical Laboratory Standards Institute (CLSI) methodologies established prior to $2011,{ }^{14}$ leading the CLSI to lower its cutoffs for susceptibility (breakpoints) for carbapenems in 2011 (Table 2). The rationale behind this decision was the desire to efficiently capture all carbapenemase producers, especially KPCs, and to avoid the routine use of the Modified Hodge Test (MHT). ${ }^{15}$ The MHT is the only test recommended by CLSI for the phenotypic detection of CRE. The MHT is currently recommended for use in microbiology laboratories that have not yet adopted the current CLSI guidelines; in these laboratories, the MHT should be performed whenever the organism is resistant to all third-generation cephalosporins and if the MIC to meropenem, imipenem, or ertapenem is $\geq 2 \mathrm{mg} / \mathrm{L} .{ }^{16}$ However, the MHT has a number of shortcomings, such as not being able to distinguish between carbapenemase types, lacking sensitivity for MBL detection, and requiring large amounts of time. ${ }^{16,17}$ A recent study by Doyle and colleagues $^{18}$ described 58\% sensitivity and 93\% specificity for identifying carbapenemases using this method.

Other phenotypic tests include inhibitor-based tests, such as EDTA and phenanthroline against MBLs, phenylboronic acid against KPCs in combination with a carbapenem in disk-diffusion, broth dilution, or the E-test. ${ }^{16,17,19}$ Other tests may detect degradation products of carbapenem hydrolysis, such as the Carba NP test or mass spectrometry, with matrix-assisted laser desorption ionization-time of flight (MALDI-TOF). ${ }^{16,20,21}$ Genotypic tests via polymerase chain reaction (PCR) are also available and can be used to detect specific resistance genes. ${ }^{16}$ Although CLSI currently does not recommend routine phenotypic or genotypic tests to confirm carbapenemase production, many of these new detection methods are promising and may play a larger role in guiding therapy with the wide dissemination of existing carbapenemases and the discovery of new carbapenemases.

In the United States, the first organism producing a KPC-type enzyme was identified in 1996 in North Carolina and has now spread to 48 states and the District of Columbia according to Centers for Disease Control and Prevention data. ${ }^{22}$ Data regarding the prevalence of CRE in pediatric patients are limited. Antimicrobial susceptibility data from The Surveillance Network Database-USA explored the prevalence of carbapenemase-producing Klebsiella pneumoniae from 2002 to 2010 from about 287 sites. The study found that by 2010, the rates of carbapenemase production among pediatric isolates was about $1.01 \% .{ }^{23}$ In addition to this report, only case reports or single-center cohort studies in pediatrics are available. Little et al $^{24}$ described 6 cases of CRE confirmed by PCR in their pediatric patient population in Seattle, Washington, between 2002 and 2010. Marchaim et $\mathrm{al}^{25}$ also described 3 cases 
Table 3. In Vitro Activity of Antimicrobial Agents Against Carbapenemase-Producing Enterobacteriaceae

\begin{tabular}{|c|c|}
\hline Antibiotic & \% Susceptible \\
\hline Ceftazidime-avibactam ${ }^{38}$ & 98 \\
\hline Colisitin 43 & 88 \\
\hline Polymyxin B ${ }^{28}$ & 93 \\
\hline Tigecycline $28,38,43$ & $91-100$ \\
\hline Meropenem ${ }^{28}$ & $20^{*}$ \\
\hline Ertapenem ${ }^{28}$ & 5 \\
\hline Piperacillin-tazobactam²8,38 & 0 \\
\hline Amikacin ${ }^{28}$ & 53 \\
\hline Gentamicin ${ }^{28,38,43}$ & $49-94$ \\
\hline Ciprofloxacin ${ }^{28}$ & 15 \\
\hline Levofloxacin ${ }^{38}$ & 7 \\
\hline Ceftriaxone 38 & 0 \\
\hline Cefepime $^{28}$ & 19 \\
\hline Aztreonam ${ }^{28}$ & 10 \\
\hline
\end{tabular}

of KPCs in pediatric patients confirmed by PCR over a 1-year period from 2008 to 2009. Lastly, a retrospective cohort study ${ }^{26}$ performed at the Children's National Medical Center in Washington, DC, from 2009 through 2011 identified 13 patients with CRE infection or colonization confirmed by the MHT.

Based on the published studies to date, healthcare exposure remains the most common risk factor for CRE acquisition. $\operatorname{Logan}^{8}$ published a review of pediatric cases of CRE from 2002 through 2010. A total of 63 cases were identified from 5 different countries, and of those cases, $87 \%$ were hospital-acquired infections. Other risk factors identified include critical illness, comorbid conditions, presence of indwelling devices, history of surgery, receipt of immunosuppressive agents, and prior antibiotic use.

\section{TREATMENT OF INFECTIONS DUE TO KPC- PRODUCING ORGANISMS}

\section{Antimicrobial Agents}

Therapeutic options for infections due to KPCproducing organisms are limited. The evidence for use of antimicrobial agents for treatment is primarily derived from in vitro and observational studies (Table 3). Data from clinical studies are scarce and are urgently needed to guide treatment decisions.

There is little evidence to support the use of carbapenem monotherapy in the treatment of KPC-producing organisms. Pooled data from Tzouvelekis et $\mathrm{al}^{19}$ showed an association between increasing carbapenem MIC and the probability of treatment failure with carbapenem monotherapy in patients infected with CRE. Treatment failure increased with MIC from $28.6 \%$ $(\mathrm{MIC}=4 \mathrm{mg} / \mathrm{L})$ to $33.3 \%(\mathrm{MIC}=8 \mathrm{mg} / \mathrm{L})$ and was as high as $75 \%$ for patients infected with an organism with an MIC of $>8 \mathrm{mg} / \mathrm{L}$. The moderate treatment success against organisms with a MIC of $\leq 8 \mathrm{mg} / \mathrm{L}$, which is considered meropenem-resistant by CLSI interpretative breakpoints, suggests carbapenems may still provide some benefit in the treatment of these extensively drug-resistant organisms, possibly in combination with other in vitro active agents such as colistin. ${ }^{27,28}$ Pharmacokinetic-pharmacodynamic (PK-PD) modeling demonstrates the potential for improved likelihood of adequate drug exposure against organisms with elevated MICs when dose optimization strategies such as high-dose, prolonged infusion regimens are employed. ${ }^{29}$

The emergence of multidrug-resistant Gram- 
negative bacteria has brought forth the resurgence of polymyxin antibiotics, polymyxin $B$ and colistin (polymyxin E), into clinical practice. Polymyxins are broad-spectrum antibiotics with activity against multidrug-resistant Gramnegative aerobic bacilli including CRE. ${ }^{30,31}$ The major dose-limiting toxicity is nephrotoxicity; approximately $18 \%$ to $61 \%$ of patients receiving polymyxins develop nephrotoxicity. ${ }^{32}$ Tigecycline is another parenterally administered, broadspectrum antibiotic with potent in vitro activity against CRE. ${ }^{30}$ Tigecycline has a large volume of distribution that leads to extensive distribution into tissue, resulting in minimal serum drug concentrations. ${ }^{33}$ This raises a substantial concern for patients with bacteremia, in whom tigecycline administration may not achieve adequate serum concentrations. The FDA issued a "Boxed Warning" citing an increase in all-cause mortality with tigecycline monotherapy compared to comparator antibiotics in a meta-analysis of Phase III and IV clinical trials. ${ }^{34}$ Finally, like all tetracyclines, there is a warning against use of tigecycline in patients younger than 8 years of age as a result of permanent tooth discoloration. Fosfomycin is an oral antibiotic used primarily in the United States for the treatment of urinary tract infections (UTIs). It is recommended by the Infectious Diseases Society of America's international clinical practice guidelines as a first-line agent for the treatment of acute uncomplicated cystitis. ${ }^{35}$ Fosfomycin has shown potent in vitro activity against MDR Gram-negative bacteria, including KPCproducing organisms. ${ }^{36}$ In a retrospective study conducted by Neuner et $\mathrm{al}^{137}$ assessing treatment of MDR UTIs, $92 \%$ of the carbapenem-resistant K pneumoniae were susceptible to fosfomycin in vitro, although only $46 \%$ showed microbiological cure.

Ceftazidime/avibactam is a broad-spectrum BLBLI combination that shows promise in the treatment of multidrug-resistant Gram-negative organisms, including carbapenem-resistant species. ${ }^{38}$ It is now FDA-approved for the treatment of complicated intra-abdominal infections (in combination with metronidazole) and complicated UTIs (including pyelonephritis) in adult patients. Because of its designation as a "Qualified Infectious Disease Product" under recent legislation aimed at speeding approval of drugs to treat resistant bacteria, its approval was based on 2 Phase II clinical trials of ceftazidime-avibactam, with Phase III trials underway (including a trial for treatment of nosocomial pneumonia). ${ }^{39-42}$ Ceftazidime-avibactam is one of the few antimicrobial agents with reliable activity against KPC-producing Gram-negative bacteria. An in vitro study ${ }^{43}$ of the agent showed good activity against meropenem-non-susceptible K pneumoniae $\left(\mathrm{MIC}_{50 / 90^{\prime}} 0.5 / 2 \mathrm{mg} / \mathrm{L}\right)$, with $98.3 \%$ of isolates $(n=115)$ susceptible to ceftazidime-avibactam (using CLSI breakpoints for ceftazidime). There are currently minimal published reports related to the use of ceftazidime/avibactam for infections due to KPC-producing bacteria, but the in vitro activity is promising and will offer a key therapeutic option to the limited arsenal, especially because of its favorable toxicity profile compared to other options.

Two new agents with activity against MDR Gram-negative bacteria are in development. Meropenem-RPX7009 is a combination of a carbapenem and a novel BLI with substantial activity against KPC-producing Enterobacteriaceae. ${ }^{44}$ Currently it is being studied in a Phase III clinical trial evaluating the efficacy compared to the best available therapy for the treatment of select serious infections due to CRE in adult patients. ${ }^{45}$ Imipenem/cilastatin-relebactam (formerly MK7655) is another antibiotic combination under development. Relebactam, a novel BLI, has in vitro data showing its ability to improve the activity of imipenem against KPC-producing Enterobacteriaceae. ${ }^{46}$ This combination is currently being studied in 2 Phase II clinical trials (complicated UTI and complicated intra-abdominal infection) in adult patients. ${ }^{47}$

\section{Monotherapy Versus Combination Therapies}

The available options lack high-quality clinical evidence regarding their efficacy for severe infections caused by KPC-producing Gram-negative bacteria. Clinical outcomes are frequently poor even when treatment is susceptible in vitro. Data from observational and case studies suggest that combination antibiotic therapy may provide some benefit over monotherapy in terms of clinical outcomes. ${ }^{48-50}$ Tumbarello et $\mathrm{al}^{48}$ conducted a multicenter, retrospective cohort study of 125 patients with KPC-producing K pneumoniae bloodstream infections treated with various monotherapy (mainly tigecycline or colistin) or 2-3-drug combination therapy regimens (mainly tigecycline/colistin, tigecycline/gentamicin, 
and tigecycline/colistin/meropenem). Median patient age in the study was around 62 years, mean APACHE III score was in the 30s, and more than $50 \%$ of patients had an unknown source of bacteremia. Death within 30 days of the first positive blood culture was the main outcome measure. There was a significantly higher likelihood of 30-day mortality in patients treated with monotherapy versus those treated with combination therapy $(54.3 \%$ versus $34.1 \% ; p=0.02)$. In logistic regression analysis, definitive treatment with a combination of tigecycline, colistin, and meropenem was associated with lower risk of mortality (odds ratio [OR] 0.11; 95\% confidence interval [CI]: 0.02-0.69; $\mathrm{p}=0.01)$. The 30-day survival rate with the combination treatment of tigecycline plus colistin was $69.7 \%$; addition of meropenem to the regimen improved survival to $87.5 \%$. Improved survival was only associated with use of a carbapenem-containing regimen in this study.

Qureshi et al ${ }^{49}$ also conducted a retrospective study assessing clinical outcomes of 41 patients with bacteremia due to KPC-producing K pneumoniae treated with monotherapy (mainly colistin-polymyxin B or tigecycline) or combination therapy (most commonly colistin-polymyxin B or tigecycline plus a carbapenem). About $40 \%$ of patients in the study were over the age of 65 years, 50\% had APACHE II scores greater than 20 , and more than $50 \%$ were admitted to the intensive care unit at enrollment. The outcome of 28-day mortality was significantly lower in the combination therapy group compared with the monotherapy group (13.3\% versus $57.8 \%$, p $=0.01$ ), although the combination therapy group had significantly more transplant patients than the monotherapy group $(53.3 \%$ versus $0 \%, \mathrm{p} \leq$ 0.001). In a multivariate analysis, combination therapy was independently associated with decreased mortality (OR 0.07; 95\% CI: 0.009-0.71; $\mathrm{p}=0.02$ ).

Daikos et $\mathrm{al}^{50}$ performed a retrospective observational study evaluating 205 patients with carbapenemase-producing K pneumoniae bloodstream infections. A majority of these patients (79.5\%) were infected with KPC or KPC/VIM. The average patient age in the study was in the 60 s; about $67 \%$ of patients met sepsis criteria, with about $20 \%$ in septic shock; and the primary source of infection was non-urinary tract sites. There was significantly higher mortality in pa- tients treated with monotherapy compared with patients treated with combination therapy $(44.4 \%$ versus $27.2 \%$; $\mathrm{p}=0.018$ ). The most common monotherapy agents were tigecycline or colistin. The lowest mortality rate $(19.3 \%)$ occurred in patients treated with a combination therapy regimen containing a carbapenem. In the Cox proportional hazards ratio model, combination therapy was associated with improved survival versus monotherapy (Hazard ratio 2.08; 95\% CI: 1.23-3.51; $\mathrm{p}=0.006$ ), which was primarily driven by carbapenem-containing combination therapy regimens. These data are consistent with those from an earlier publication ${ }^{27}$ that compiled several case reports, case series, retrospective studies, and one prospective observational study and concluded that carbapenems may have activity against KPCs with an MIC of $\leq 4 \mathrm{mg} / \mathrm{L}$ and that this activity may be enhanced with large-dose prolonged infusion carbapenems (to optimize PK-PD target attainment) in combination with another in vitro active agent.

Paul et al $^{51}$ completed a meta-analysis of 16 studies and case series comparing colistin monotherapy versus colistin-based combination therapy for treatment of infections due to carbapenemase-producing or cabapenemaseresistant Gram-negative bacteria. There were no randomized controlled trials assessing the treatment outcomes of KPC-producing bacteria. The pooled analysis of colistin versus colistincombination (carbapenem, tigecycline, or aminoglycoside) therapy did not show a difference in mortality. Tzouvelekis et $\mathrm{al}^{19}$ also performed a literature review and consolidation of data from low-quality evidence primarily composed of observational studies and case series. A total of 161 patients were identified with infections due to KPC-producing K pneumoniae, which included bloodstream infections as the majority. The composite analysis included an additional 140 patients infected with MBL-producing $K$ pneumoniae. Treatment failure rates were assessed in reference to 7 treatment regimen groups: combination therapy with $\geq 2$ active drugs (one being a carbapenem), combination therapy with $\geq 2$ active drugs (no carbapenem), carbapenem monotherapy, aminoglycoside monotherapy, tigecycline monotherapy, colistin monotherapy, and, lastly, inappropriate therapy. The lowest failure rate $(8.3 \%)$ was observed in the carbapenem combination therapy group. On the other end of 
the spectrum, monotherapy with tigecycline or colistin had comparable failure rates to patients categorized as receiving inappropriate therapy.

\section{Considerations for Pediatrics}

Data for the treatment of KPC-producing organisms are limited in adults, but even more so in the pediatric population. A recent review of the literature by Logan ${ }^{8}$ identified 6 studies that included 63 children with infection due to CRE. The cases had a worldwide geographic distribution with only $11 \%$ from the United States. There were 24 cases with data on antibiotic treatment, with the majority of children receiving aminoglycosides as either monotherapy or as part of combination therapy. Children in the study were typically very young, critically ill, and had additional risk factors, such as immunosuppressive therapy and prior antibiotic use. At this time, there are no well-controlled clinical trials to serve as high-quality evidence for treatment of KPCproducing organisms in pediatrics. Currently, clinicians treating pediatric patients are mainly guided by data from observational studies in the adult population.

Dosing in children is an important consideration in order to maximize the effectiveness of the limited treatment options. Unfortunately, few data exist to guide dosing in younger children. A Monte Carlo simulation study by Courter et $\mathrm{al}^{28}$ demonstrated that the use of large-dose prolonged infusion carbapenems (meropenem $40 \mathrm{mg} / \mathrm{kg} /$ dose intravenously every 8 hours infused over 3 hours) improves the likelihood of achieving adequate drug exposure $(40 \% \mathrm{~T}>\mathrm{MIC})$ for an MIC of $8 \mathrm{mg} / \mathrm{L}$ as compared to a standard 30 -minute infusion, with $97 \%$ versus $33 \%$ target attainment, respectively. Dosing of colistin in pediatrics is mainly guided by the manufacturer recommendations of a dose of $2.5 \mathrm{mg} / \mathrm{kg} /$ dose of colistin base activity (CBA) intravenously every 12 hours..$^{52}$ There are no formal studies assessing the use of a loading dose, but several investigators ${ }^{53}$ have suggested the use of a loading dose ( $5 \mathrm{mg} / \mathrm{kg}$ of CBA) in order to achieve target serum drug concentration more rapidly. The use of tigecycline in pediatrics is limited by toxicity concerns, and dosing recommendations are based on pharmacokinetic studies (8-11 years: $1.2 \mathrm{mg} / \mathrm{kg} /$ dose every 12 hours with a maximum of $50 \mathrm{mg}$ per dose; $12-17$ years: $50 \mathrm{mg}$ every 12 hours). ${ }^{53}$ At this time, there are no clinical data or dosing guidance for ceftazidime-avibactam in pediatric patients, although there is an ongoing Phase I study ${ }^{54}$ of ceftazidime-avibactam in pediatrics assessing the pharmacokinetics, safety, and tolerability of a single dose in children from 3 months to 18 years of age.

\section{CONCLUSIONS}

Once a concerning but rare phenomenon, carbapenemase-producing organisms have developed into a serious public health threat. Although to date there have been relatively few infections in children, as CRE becomes endemic in healthcare settings the risks of spread into the pediatric population are substantial. The relationships between in vitro susceptibility testing and clinical outcomes for these organisms are only beginning to be definitively studied. For example, despite in vitro resistance, there may be a role for aggressively dosed carbapenems as part of combination therapy regimens. Unfortunately, few data exist to guide treatment recommendations or dosing in children. One new agent with potent activity against KPC-producing organisms has been recently approved, and some promising agents are in late-stage development. It will take these efforts and more to avoid the prospect of a "new normal" wherein common bacterial pathogens like Klebsiella routinely cause untreatable infections.

Disclosure The authors declare no conflicts or financial interest in any product or service mentioned in the manuscript, including grants, equipment, medications, employment, gifts, and honoraria.

Abbreviations BLBLI, beta-lactam-beta-lactamase inhibitor; BLI, beta-lactamase inhibitor; CBA, colistin base activity; $\mathrm{Cl}$, confidence interval; $\mathrm{CLSI}$, Clinical Laboratory Standards Institute; CRE, carbapenem-resistant Enterobacteriaceae; EDTA, ethylenediamine tetraacetic acid; FDA, Food and Drug Administration; IMP, active on imipenem; KPC, Klebsiella pneumoniae carbapenemase; $\mathrm{MBL}$, metallo-betalactamase; MHT, Modified Hodge Test; MIC, minimum inhibitory concentration; NDM, New Delhi metallo-betalactamase; OR, odds ratio; OXA-48, oxacillin-hydrolyzing; PCR, polymerase chain reaction; PK-PD, pharmacokineticpharmacodynamic; UTI, urinary tract infection; VIM, Verona integron-encoded metallo-beta-lactamase

Correspondence Conan MacDougall, PharmD, MAS, 521 Parnassus Avenue, C-152 Box 0622, San Francisco, CA 94143-0622, email: conan.macdougall@ucsf.edu 


\section{REFERENCES}

1. Hersh AL, Shapiro DJ, Pavia AT, Shah SS. Antibiotic prescribing in ambulatory pediatrics in the United States. Pediatrics. 2011;128(6):1053-1061.

2. Queenan AM, Bush K. Carbapenemases: the versatile beta-lactamases. Clin Microbiol Rev. 2007;20(3):440-458.

3. Centers for Disease Control and Prevention. Threat Report 2013. http:/ / www.cdc. gov/drugresistance/threat-report-2013/. Accessed January19, 2016.

4. Papp-Wallace KM, Endimiani A, Taracila MA, Bonomo RA. Carbapenems: past, present, and future. Antimicrob Agents Chemother. 2011;55(11):4943-4960.

5. Livermore DM. Beta-lactamases in laboratory and clinical resistance. Clin Microbiol Rev. 1995;8(4):557.

6. Curello J, MacDougall C. Beyond susceptible and resistant, part II: treatment of infections due to Gram-negative organisms producing extended-spectrum $\beta$-lactamases. J Pediatr Pharmacol Ther. 2014;19(3):156-164.

7. Crandon JL, Nicolau DP. Human simulated studies of aztreonam and aztreonam-avibactam to evaluate activity against challenging Gram-negative organisms, including metallo- $\beta$-lactamase producers. Antimicrob Agents Chemother. 2013;57(7):3299-3306.

8. Logan LK. Carbapenem-resistant Enterobacteriaceae: an emerging problem in children. Clin Infect Dis. 2012;55(6):852-859.

9. Aktaş Z, Kayacan C, Oncul O. In vitro activity of avibactam (NXL104) in combination with $\beta$-lactams against Gram-negative bacteria, including OXA-48 $\beta$-lactamaseproducing Klebsiella pneumoniae. Int J Antimicrob Agents. 2012;39(1):86-89.

10. Ehmann DE, Jahić H, Ross PL, et al. Kinetics of avibactam inhibition against Class A, C, and D B-Lactamases. J Biol Chem. 2013;288(39):27960-27971.

11. Endimiani A, Hujer KM, Hujer AM, et al. Evaluation of ceftazidime and NXL104 in two murine models of infection due to KPCproducing Klebsiella pneumoniae. Antimicrob Agents Chemother. 2011;55(1):82-85.
12. Castanheira M, Farrell SE, Deshpande LM, et al. Prevalence of $\beta$-lactamase-encoding genes among Enterobacteriaceae bacteremia isolates collected in 26 U.S. hospitals: report from the SENTRY antimicrobial surveillance program (2010). Antimicrob Agents Chemother. 2013;57(7):3012-3020.

13. Cantón R, Canut A, Morosini MI, Oliver A. Breakpoints for carbapenemase-producing Enterobacteriaceae: is the problem solved? Enfermedades Infecc Microbiol Clín. 2014;32(suppl 4):33-40.

14. Clinical and Laboratory Standards Institute (CLSI). Performance Standards for Antimicrobial Susceptibility Testing; 19th Informational Supplement: CLSI document M100-S19. Wayne, PA: CLSI; 2009.

15. Hrabák J, Chudáčková E, Papagiannitsis CC. Detection of carbapenemases in Enterobacteriaceae: a challenge for diagnostic microbiological laboratories. Clin Microbiol Infect. 2014;20(9):839-853.

16. Clinical and Laboratory Standards Institute. Performance Standards for Antimicrobial Susceptibility Testing; 25th Informational Supplement: CLSI document M100-S25. Wayne, PA: CLSI; 2015.

17. Nordmann P, Poirel L. Strategies for identification of carbapenemase-producing Enterobacteriaceae. J Antimicrob Chemother. 2013;68(3):487-489.

18. Doyle D, Peirano G, Lascols C, et al. Laboratory detection of Enterobacteriaceae that produce carbapenemases. J Clin Microbiol. 2012;50(12):3877-3880.

19. Tzouvelekis LS, Markogiannakis A, Psichogiou M, et al. Carbapenemases in Klebsiella pneumoniae and other Enterobacteriaceae: an evolving crisis of global dimensions. Clin Microbiol Rev. 2012;25(4):682-707.

20. Nordmann P, Poirel L, Dortet L. Rapid detection of carbapenemase-producing Enterobacteriaceae. Emerg Infect Dis. 2012;18(9):1503-1507.

21. Dortet L, Poirel L, Nordmann P. Rapid identification of carbapenemase types in Enterobacteriaceae and Pseudomonas spp. by using a biochemical test. Antimicrob Agents Chemother. 2012;56(12):6437-6440. 
22. Munoz-Price LS, Quinn JP. The spread of Klebsiella pneumoniae carbapenemases: a tale of strains, plasmids, and transposons. Clin Infect Dis. 2009;49(11):1739-1741.

23. Braykov NP, Eber MR, Klein EY, et al. Trends in resistance to carbapenems and third-generation cephalosporins among clinical isolates of Klebsiella pneumoniae in the United States, 1999-2010. Infect Control Hosp Epidemiol. 2013;34(3):259-268.

24. Little ML, Qin X, Zerr DM, Weissman SJ. Molecular diversity in mechanisms of carbapenem resistance in paediatric Enterobacteriaceae. Int J Antimicrob Agents. 2012;39(1):52-57.

25. Marchaim D, Chopra T, Bhargava A, et al. Recent exposure to antimicrobials and carbapenem-resistant Enterobacteriaceae: the role of antimicrobial stewardship. Infect Control Hosp Epidemiol. 2012;33(8):817-830.

26. Dirajlal-Fargo S, DeBiasi R, Campos J, Song $X$. Carbapenem-resistant Enterobacteriaceae in pediatric patients: epidemiology and risk factors. Infect Control Hosp Epidemiol. 2014;35(4):447-449.

27. Daikos GL, Markogiannakis A. Carbapenemase-producing Klebsiella pneumoniae: (when) might we still consider treating with carbapenems? Clin Microbiol Infect. 2011;17(8):1135-1141.

28. Courter JD, Kuti JL, Girotto JE, Nicolau DP. Optimizing bactericidal exposure for beta-lactams using prolonged and continuous infusions in the pediatric population. Pediatr Blood Cancer. 2009;53(3):379-385.

29. Kuti JL, Dandekar PK, Nightingale CH, Nicolau DP. Use of Monte Carlo simulation to design an optimized pharmacodynamic dosing strategy for meropenem. JClin Pharmacol. 2003;43(10):1116-1123.

30. Castanheira M, Sader HS, Deshpande LM, et al. Antimicrobial activities of tigecycline and other broad-spectrum antimicrobials tested against serine carbapenemase- and metallo-\{beta\}-lactamase-producing Enterobacteriaceae: report from the SENTRY antimicrobial surveillance program. Antimicrob Agents Chemother. 2008;52(2):570-573.
31. Gales AC, Jones RN, Sader HS. Contemporary activity of colistin and polymyxin B against a worldwide collection of Gram-negative pathogens: results from the SENTRY Antimicrobial Surveillance Program (2006-09). J Antimicrob Chemother. 2011;66(9):2070-2074.

32. Justo JA, Bosso JA. Adverse reactions associated with systemic polymyxin therapy. Pharmacotherapy. 2015;35(1):28-33.

33. Barbour A, Schmidt S, Ma B, et al. Clinical pharmacokinetics and pharmacodynamics of tigecycline. Clin Pharmacokinet. 2009;48(9):575-584.

34. Dixit D, Madduri RP, Sharma R. The role of tigecycline in the treatment of infections in light of the new black box warning. Expert Rev Anti Infect Ther. 2014;12(4):397-400.

35. Gupta K, Hooton TM, Naber KG, et al. International clinical practice guidelines for the treatment of acute uncomplicated cystitis and pyelonephritis in women: a 2010 update by the Infectious Diseases Society of America and the European Society for Microbiology and Infectious Diseases. Clin Infect Dis. 2011;52(5):e103-e120.

36. Reffert JL, Smith WJ. Fosfomycin for the treatment of resistant Gram-negative bacterial infections. Pharmacother J Hum Pharmacol Drug Ther. 2014;34(8):845-857.

37. Neuner EA, Sekeres J, Hall GS, van Duin D. Experience with fosfomycin for treatment of urinary tract infections due to multidrug-resistant organisms. Antimicrob Agents Chemother. 2012;56(11):5744-5748.

38. Lagacé-Wiens P, Walkty A, Karlowsky J. Ceftazidime \& avibactam: an evidencebased review of its pharmacology and potential use in the treatment of Gramnegative bacterial infections. Core Evid. 2014:13.

39. Press Announcements. FDA Approves New Antibacterial Drug Avycaz. http:/ / www.fda. gov/NewsEvents/Newsroom/PressAnnouncements/ucm435629.htm. Accessed January 19, 2016.. 
40. Lucasti C, Popescu I, Ramesh MK, et al. Comparative study of the efficacy and safety of ceftazidime/avibactam plus metronidazole versus meropenem in the treatment of complicated intra-abdominal infections in hospitalized adults: results of a randomized double-blind, Phase II trial. J Antimicrob Chemother. 2013;68(5):1183-1192.

41. Vazquez JA, Gonzalez Patzan LD, Stricklin D, et al. Efficacy and safety of ceftazidimeavibactam versus imipenem-cilastatin in the treatment of complicated urinary tract infections, including acute pyelonephritis, in hospitalized adults: results of a prospective, investigator blinded, randomized study. Curr Med Res Opin. 2012;28(12):19211931.

42. ClinicalTrials.gov. A Study Comparing Ceftazidime-Avibactam Versus Meropenem in Hospitalized Adults With Nosocomial Pneumonia. https: / / clinicaltrials.gov/ct2/show/ NCT01808092?term=ceftazidime+avibact am\&rank=2. Accessed January 19, 2016.

43. Sader HS, Castanheira M, Flamm RK, et al. Antimicrobial activity of ceftazidimeavibactam against Gram-negative organisms collected from U.S. medical centers in 2012. Antimicrob Agents Chemother. 2014;58(3):1684-1692.

44. Sabet M, Tarazi Z, Nolan T, et al. In vivo efficacy of carbavance (meropenem/rpx7009) against KPC-producing Enterobacteriaceae: Proceedings of the 54th International Conference on Antimicrobial Agents and Chemotherapy, Washington, DC, 2014.

45. ClinicalTrials.gov. Efficacy, Safety, Tolerability of Carbavance Compared to Piperacillin/ Tazobactam in cUTIs, Including AP, in Adults. https: / / clinicaltrials.gov / ct2 / show / NCT02166476. Accessed January 2016.

46. Livermore DM, Warner M, Mushtaq S. Activity of MK-7655 combined with imipenem against Enterobacteriaceae and Pseudomonas aeruginosa. J Antimicrob Chemother. 2013;68(10):2286-2290.
47. ClinicalTrials.gov. Study of the Safety, Tolerability, and Efficacy of MK-7655 + Imipenem/Cilastatin Versus Imipenem/Cilastatin Alone for the Treatment of Complicated Urinary Tract Infection (cUTI) (MK-7655-003). https://www.clinicaltrials.gov/ct2/show/ NCT01505634. Accessed January 2016.

48. Tumbarello M, Viale P, Viscoli C, et al. Predictors of mortality in bloodstream infections caused by Klebsiella pneumoniae carbapenemase-producing K. pneumoniae: importance of combination therapy. Clin Infect Dis. 2012;55(7):943-950.

49. Qureshi ZA, Paterson DL, Potoski BA, et al. Treatment outcome of bacteremia due to KPC-producing Klebsiella pneumoniae: superiority of combination antimicrobial regimens. Antimicrob Agents Chemother. 2012;56(4):2108-2113.

50. Daikos GL, Tsaousi S, Tzouvelekis LS, et al. Carbapenemase-producing Klebsiella pneumoniae bloodstream infections: lowering mortality by antibiotic combination schemes and the role of carbapenems. Antimicrob Agents Chemother. 2014;58(4):23222328.

51. Paul M, Carmeli Y, Durante-Mangoni E, et al. Combination therapy for carbapenemresistant Gram-negative bacteria. J Antimicrob Chemother. 2014:69(9):2305-2309.

52. Monarch Pharmaceuticals. Coly-Mycin $M$ Parenteral [package insert]. Bristol, TN: Monarch Pharmaceuticals; 2002.

53. Hsu AJ, Tamma PD. Treatment of multidrug-resistant Gram-negative infections in children. Clin Infect Dis. 2014;58(10):14391448.

54. ClinicalTrials.gov. Safety and Tolerability of Ceftazidime-Avibactam for Pediatric Patients With Suspected or Confirmed Infections. https:/ / www.clinicaltrials.gov/ct2/show / NCT01893346?term $=$ ceftazidime+avibacta $\mathrm{m}+$ pediatric\&rank=1. Accessed January 19, 2016. 